\title{
Measuring the stare-in-the-crowd effect: a new paradigm to study social perception
}

\author{
Eileen T. Crehan • Robert R. Althoff
}

Published online: 21 November 2014

(C) Psychonomic Society, Inc. 2014

\begin{abstract}
Social perceptual ability plays a key role in successful social functioning. Social interactions demand a number of simultaneous skills, one of which is the detection of self-directed gaze. This study demonstrates how the ability to accurately detect self-directed gaze, called the stare-in-thecrowd effect, can be studied using a new eye-tracking paradigm. A set of images was developed to test this effect using a group of healthy undergraduate students. Eye movements and pupil size were tracked while they viewed these images. Participants also completed behavioral measures about themselves. Results show that self-directed gaze results in significantly more looking by participants. Behavioral predictors of gaze behaviors were not identified, likely given the health of the sample. However, correlations with variables are reported to explore in future research.
\end{abstract}

Keywords Stare-in-the-crowd effect · Eye-tracking · Eye contact

\section{Introduction}

Humans of all ages are almost constantly viewing faces. At work, we determine when to jump in on water cooler conversations by initiating eye contact or following the gaze of others. On the commuter bus, your newspaper reading may be interrupted by that vague feeling that someone is looking at you, only to look up as a person suddenly shifts their gaze elsewhere. During recess, the direction of a ringleader's gaze indicates whether they are inviting you to play or if you are

\section{E. T. Crehan $\cdot$ R. R. Althoff $(\bowtie)$}

Department of Psychiatry, Vermont Center for Children, Youth, and Families at the University of Vermont, 364 SJ3 FAHC, 1 S. Prospect, Burlington, VT 05401, USA

e-mail: ralthoff@uvm.edu being excluded. The volume of experiences involving gaze detection and scanning of faces is quite large and each experience informs our social processing and decision making. Despite the pervasive use of these skills, there has been extremely limited study of the mechanisms of gaze detection in groups and how shifting gaze affects the looker. This study was designed to capture this critical phenomenon using an eye-tracking paradigm. Once a model of this experience is developed, targeted interventions can be designed to help those who struggle with this cornerstone of social activity.

Gaze is a key tool in initiating, sustaining, and ending social interactions. When someone is looking at us (selfdirected gaze), our own gaze is attracted back (Farroni, Csibra, Simion, \& Johnson, 2002; Kleinke, 1986). Compared to gaze directed elsewhere, self-directed gaze has been shown repeatedly to be more interesting, as indexed by longer looking times. This ability to quickly pick out selfdirected gaze from a group of faces is referred to as the "starein-the-crowd" effect (SITC). That humans are programmed to preferentially notice self-directed gaze is likely a function of the important role of gaze in social functioning. Human response to others looking at them is generally positive (except if the gaze is threatening or competitive) (Jellison \& Ickes, 1974; Kleinke, 1986). Maintaining eye contact during social interactions has been shown to have a range of implications, including being perceived as more attentive (Breed, 1972; Kelly, 1978), intelligent (Wheeler, Baron, Michell, \& Ginsburg, 1979), and pleasant (Cook \& Smith, 1975).

Simply staring at others, however, does not assure a successful social interaction. In most cases, both inconsistent and constant eye contact result in negative perceptions while well modulated and frequent eye contact result in positive perceptions. Thus, dynamic gaze abilities are in fact predictive of successful social interactions. Eye contact provides critical information about next steps in the interaction; in the passing back and forth of the proverbial "talking stick" in the course of 
a conversation, longer gazes are used to convey that a speaker is finishing what they are saying and waiting for someone else to respond (Kendon, 1967; Levine \& Sutton-Smith, 1973). Similarly, breaking eye contact has been shown to indicate the end of a social interaction (Knapp, Hart, Friedrich, \& Shulman, 1973). These results generalize to other broader settings, such as the classroom. For instance, it has been found that learning is enhanced when taught by a gazing teacher and that seating arrangements in which mutual gaze is possible facilitates cooperative interactions (Jellison \& Ickes, 1974; Otteson \& Otteson, 1980).

Once gaze is detected, other advanced social processes begin. The stare-in-the-crowd effect serves a vital role by initiating quick access to social information about others and allowing for emotion identification. Making eye contact allows for faster access to stored, categorical social information such as gender and race (Macrae \& Bodenhausen, 2000). Shorter times to access this information ease interaction, as past experiences or social information inform a current interaction. Macrae et al. (2002) assessed the interaction of gaze and access to categorical social information. They found that participants were significantly faster at categorizing targets in photographs as male or female when the targets were looking straight ahead with open eyes, similar to "direct gaze." Furthermore, Macrae et al. used similar images flashed briefly before stereotypically male or female words and found that words were sorted significantly faster when the flashed target was looking straight ahead. It appears, therefore, that accessing this information is crucial to correctly interpret social cues such as gender and race.

As adults, internal features of the face (i.e., the eyes, nose, and mouth) are looked at more than external features such as hair, forehead, or ears (Walker-Smith, Gale, \& Findlay, 1977; Yarbus, 1967). Even though internal features are more often looked at, the eyes themselves play a key role in perception of others. In fact, if the eye region is covered up, one's ability to recognize a face diminishes significantly, a shift that does not occur when the mouth or nose is obscured (McKelvie, 1976). The ability to detect and reciprocate direct gaze thus also has implications for accessing social information.

Despite the ease of capturing gaze behavior, there is much work to be done to fill in a comprehensive social perception model of dynamic gaze. Detection of self-directed gaze has historically been studied using single-sitter photographs (von Grunau \& Anston, 1995) and, more recently, video clips of dyads (Klin, Jones, Schultz, Volkmar, \& Cohen, 2002) with little to know attention to the situation of multiple sitters presented in each image. Additionally, two commonplace conditions, "getting caught staring" and "catching another staring" are barely reviewed in the literature. These two phenomena are integral to understanding the events that make up the timeline of initiating and ending mutual gaze (i.e., two people looking at one another). Getting caught staring is an important social tool, especially when used intentionally. Staring for a short period of time can open up a conversation or serve as a way to meet new people. In other situations, getting caught staring may be socially inappropriate. There is a need for a paradigm to measure these two critical components of social interaction and the aim of the current study was to establish such a paradigm.

Here, four main phenomena are examined: (1) direct gaze, to directly measure the SITC effect; (2) withdrawn gaze, to measure how one scans a group of faces when none are selfdirected; (3) "getting caught staring," to present a dynamic scene in which a previously averted face becomes selfdirected as a participant scans their face; (4) "catching another staring," to present another dynamic scene in which a selfdirected face suddenly shifts their gaze elsewhere in response to eye contact from the participant. The concept of "getting caught staring" is modeled using a condition where selfdirected gaze occurs once participants fixate on an initially averted target face. The concept of "catching someone else staring" is modeled using a condition where once participants fixate on a target face with self-directed gaze, the target looks away. To the best of the authors' knowledge, these components of the stare-in-the-crowd effect have not been explored in the literature.

In this study, a range of behavioral measures were included to augment the data obtained from the visual paradigm. These measures quantify social responsiveness, social anxiety, adaptive functioning, and psychiatric syndromes. Social responsiveness and adaptive functioning were hypothesized to be positively correlated with increased looking at images and that symptoms of social anxiety would predict less looking, especially in the dynamic conditions. Syndromes such as aggressive problems and attention were predicted to be, respectively, positively correlated and negatively correlated with looking. Across conditions, self-directed gaze was predicted to attract more eye gaze (quantified as longer dwell times and more fixations) and that changes in gaze, such as in the getting caught staring and catching another staring conditions, would also attract more looking from participants. Previous research has indicated that pupil dilation indicates surprise or interest (Preuschoff, Hart, \& Einhauser, 2011). Pupil dilation is also shown to occur when attention is sustained and the brain is processing information (Steinhauer, Siegle, Condray, \& Pless, 2004). Thus, conditions in which the images shift or in which there is self-directed gaze were hypothesized to also result in more pupil dilation.

\section{Methods}

Sample Participants were undergraduate students in an introductory psychology course. An announcement was distributed to classes in which students could earn class credit for 
participating in a research study. Inclusion criteria included having normal or corrected-to-normal vision, no known diagnosis of a pervasive developmental disorder or social anxiety, and no current psychotropic medications. A total of 35 students (20 female) participated with a mean age of 21 years $(\mathrm{SD}=0.81)$. This study was approved by the Institutional Review Board at the University of Vermont. Written consent was obtained from all participating adults.

Procedure Participants were consented and seated in front of the eye-tracking camera with their head resting on a chin rest. Four blocks were presented with 35 images in each block. Only the stable averted or stable self-directed images were presented in the first block to acclimate the participants to the task. In blocks two through four, images from each of the four conditions were presented randomly. The blocks were counterbalanced across participants.

Photographs To validate the stare-in-a-crowd effect, visual stimuli developed for the purpose of this project were presented. In the literature, studies typically rely on stimulus photos of individuals or sketches of pairs of eyes. This does not allow for assessment of gaze behavior in realistic situations (Fletcher-Watson, Leekam, Benson, Frank, \& Findlay, 2009). Using a group of faces instead of an individual (Fig. 1) facilitates the translation of results into actual social situations (Klin et al., 2002). There were four stimulus conditions as described in the hypothesis section for the photographs: stable self-directed; (2) delayed self-directed; (3) stable averted; (4) delayed averted (Fig. 2).

The sophistication and sensitivity of eye-tracking technology is most clearly apparent in the "delayed" conditions. Here, "trigger boundaries" were drawn around faces in the photo. Once the subject directed his or her gaze at a particular face, i.e., within the trigger boundary, this cued a change in gaze (Fig. 3). For instance, in the delayed self-directed condition, if the subject looks in the eye boundary of Person A with averted gaze, this will instantly trigger a new image in which the eyes of Person A are now subject- ('self-') directed. Similarly, for the delayed averted condition, the subject will look in the eye boundary of Person A who is exhibiting subject- (or self-) directed gaze and this will trigger a new image in which the eyes of Person A are now averted.
Characteristics of images The stimuli used in this pilot study were developed with the goal of creating a set of stimuli to be used again in the future. Thus, a range of photographs with varying combinations of targets were included. Number of targets in the images ranged from 9 to twenty-seven. Most of the images (about $95 \%$ ) had an even number of male and female targets and the remaining images were all male or all female. Targets were told to display neutral facial expressions and images in which targets were obviously displaying emotion (as rated by the authors and confirmed by research assistants) were not included in the presented stimuli. Additionally, some images featured close up faces while others were taken from farther away, in a classroom setting. Although comparisons of behavior relative to stimuli characteristics were not made for the purposes of this pilot study, such manipulations will be possible in the future.

Presentation timing For the stable conditions, images were presented for 16 seconds (for consistency with the dynamic conditions, the eight-second mark was considered the onset of the second image, even though the image remained the same). For the dynamic scenes, the initial image was presented for eight seconds or when the trigger boundary was crossed, whichever came first, and the second image was then present for eight seconds.

Eyetracker The EyeLink1000 collects over 100 measures of gaze behavior, the term used here to refer to eye movements such as fixation location, fixation duration, saccades, blinks, etc. The sensitivity of the camera allows for detection of eye movements as small as 0.5 degrees of visual angle. Sitting 18 inches from a computer monitor, this translated into 0.2 inches on the monitor. Given the images used here, this allowed for differentiation between a glance at the left versus right eye. Preset areas of interest allowed for comparisons of time spent looking at different regions of an image, for instance the eyes versus non-eye facial regions. This quantitative monitoring of these fixation locations, fixation durations, and looking patterns conveys inner processes of the viewer.

Data Cleaning Since these images were being used for the first time in this study, inspection of the data demonstrated that some images were more reliable across participants than

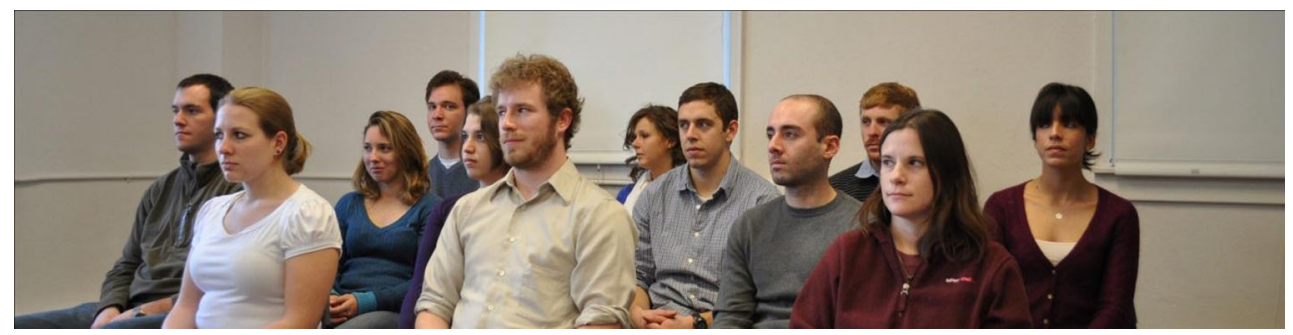

Fig. 1 An example of the visual stimuli developed for this study. In each image, either one stimulus face is self-directed or none are self-directed, i.e. averted gaze 
Withdrawn and direct gaze: the stable averted and stable self-

directed conditions were

compared

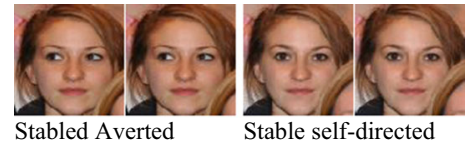

Catching someone else staring: The delayed averted condition was compared to both stable averted (withdrawn) and stable self-

directed (direct) gaze to isolate the specific effects of gaze that shifts away from a participant once eye contact is made.

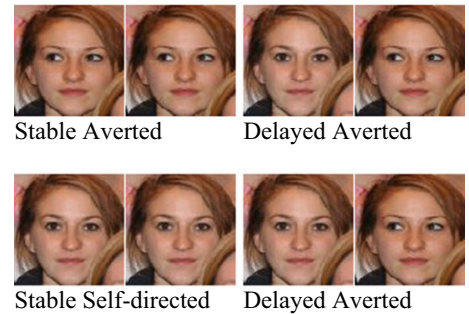

\section{Getting caught staring:}

The delayed self-directed

condition was compared to both stable averted (withdrawn) and

stable self-directed (direct) gaze to isolate the specific effects of gaze that shifts toward a participant currently looking at someone else.

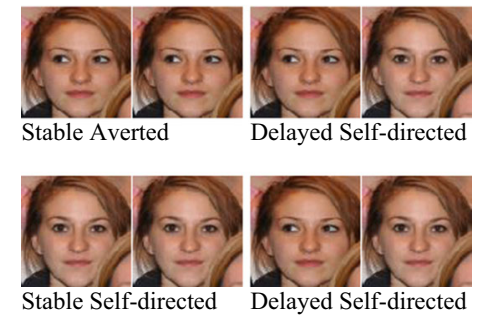

Fig. 2 Example of gaze condition comparisons. For the "delayed" conditions, fixation on the eyes of the first picture triggers the change in gaze of the second image. In the "stable conditions," fixation on the face does not result in shifting gaze

others. A large number of stimuli were presented to participants with the goal of separating out high quality visual stimuli (i.e., images in which trigger boundaries were consistently crossed) and lower quality. For instance, some images had a target sitter whose eyes were obstructed by his or her hair. As evidenced by the eye-tracking results, these were situations where the eyes were difficult to detect and thus images in which this type of target was sitting were excluded. Additionally, if an image resulted in missing data for more than $75 \%$ of the participants (e.g., crossing the trigger boundary did not lead to a change in the picture), that image was not included in the analysis. Of the 95 images used in the study, $37 \%$ of original images were excluded from analyses due to missing data across participants. The remaining images demonstrated utility in measuring the SITC effect; these images comprise the set of visual stimuli used in the analysis.

Data Preparation For each participant, each eye-tracking outcome was averaged by condition. For instance, IA Dwell Time was averaged for participant \#1 for each of the stable self-directed images they viewed. These averages were used to make comparisons between conditions.

Statistical Analysis Analysis of variance (ANOVA) procedures were used to identify condition differences on the eyetracking measures using SPSS 19.0. To compare direct and withdrawn gaze, comparisons between the stable self-directed and stable averted conditions were made. To explore the
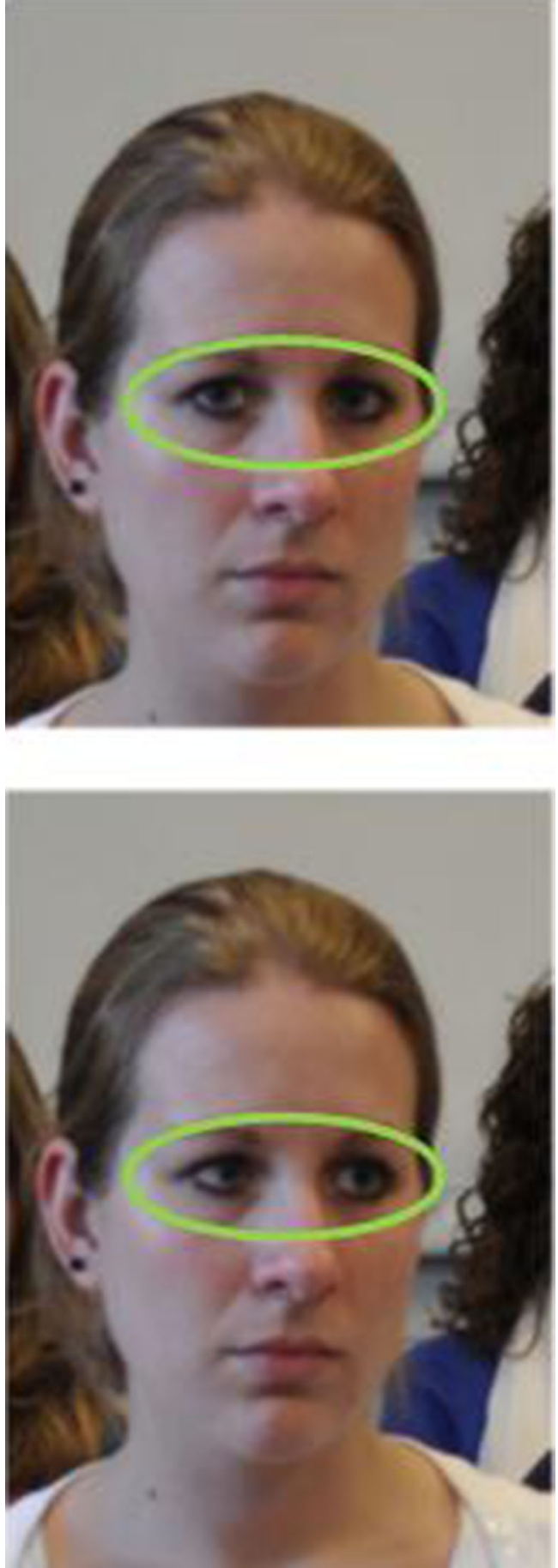

Fig. 3 The top face illustrates "selfdirected" gaze. The bottom face illustrates "averted" gaze. The ovals are boundaries used for analytic purposes

"catching someone else staring" phenomenon, the delayed averted condition was compared to both the stable averted and stable self-directed conditions. To explore the "being caught staring" phenomenon, the delayed self-directed condition was compared to both the stable averted and stable selfdirected conditions. Comparisons against both "stable" conditions were made to illustrate the effects of withdrawn or 
direct gaze in relation to the shifting gaze conditions. Eta squared formulas were used to calculate effect size.

In addition to comparing average pupil size across conditions, additional analyses were conducted to examine pupil changes within condition. According to Beatty (1982), peak pupil dilation occurs between 1,800 and 2,000 milliseconds after stimulus onset. An average pupil size was calculated from the first five fixations of each image, resulting in two averages per visual presentation, to insure that this peak dilation in reaction to change was included in the interval. For instance, in the Delayed SelfDirected condition, pupil diameter from the first five fixations in the averted image was calculated, and then again from the first five fixations in the direct image. Z-score transformations were calculated for each participant for each image, providing a standardized measure of pupil diameter change relative to the individual.

\section{Behavioral measures}

A summary of the scales and items used is provided in the descriptives table (Table 1).

The Liebowitz Social Anxiety Scale (LSAS) (Heimberg et al., 1999) is a highly reliable $(\alpha=.96) 24$-item measure of social anxiety. Stable self-directed versus stable averted (Table 2): 0-3 for Fear or Anxiety and from 0-3 for Avoidance. Item number 19 ("Looking at people you don't know very well in the eyes") was isolated for use in analyses described below due to its relevance to the paradigm.

The Social Responsiveness Scale - Adult Self-Report (SRS-ASR) (Constantino \& Gruber, 2005) is a 65-item measure of the frequency of social response. The SRS-ASR has a

Table 1 Means and SD for behavioral measures

\begin{tabular}{llll}
\hline & & Mean & SD \\
\hline Adult Self Report & Aggressive behavior & 53.67 & 5.12 \\
& Attention problems & 56.94 & 6.02 \\
& Internalizing & 52.39 & 12.43 \\
& Externalizing & 50.00 & 10.75 \\
& Adaptive Functioning- & 54.81 & 5.74 \\
Liebowitz Social & Friends & & \\
Anxiety Scale & Item \#19: Fear & 0.63 & 0.87 \\
Social Responsiveness & Item \#19: Avoidance & 0.63 & 0.88 \\
Scale- Adult Self & Cognitive & 18.91 & 3.50 \\
Report & Expressive & 35.72 & 8.17 \\
& Social & 20.62 & 5.68 \\
& Preoccupations & 19.03 & 4.78 \\
& Receptive & 15.41 & 2.29 \\
& Total & 91.62 & 45.79 \\
\hline
\end{tabular}

Cronbach's $\alpha$ of .71 in typically developing populations (Bolte, 2012). Respondents rate each item on a 1 (Not True) to 4 (Almost Always True) scale. A total of five subscale (Receptive, Social, Expressive Language, Cognitive, Preoccupations) scores are calculated. The presence of an ASD is suggested by a high total score on this measure. Although the SRS is not a diagnostic tool, it has utility in identifying adults with profiles similar to those who have an autism spectrum disorder (ASD).

The Adult Self Report (ASR) (Achenbach, 2009) is a measurement tool from the Achenbach system of measurement that is completed by adults about themselves. The 126-item measure surveys for psychopathology and functional impairment. For the subscales used in the following analyses (Aggressive Behavior, Attention Problems, Internalizing Problems, Externalizing Problems, Adaptive Functioning-Friends), Cronbach's $\alpha$ ranged from .69 (Adaptive Functioning-Friends) to .93 (Internalizing Problems) for both referred and non-referred populations. Given the findings between different types of psychopathology (i.e., aggressive behaviors, attention) and gaze behavior, this measure was included to survey for a range of possible psychopathology.

Pearson's correlations between the five eye-tracking variables used above were run with specific scales or items. From the ASR, Aggressive Behavior, Attention Problems, Internalizing Problems, Externalizing Problems, and Adaptive Functioning-Friends were included. Each of the subscales from the SRS as well as the total score were included. From the LSAS, the Fear and Avoidance ratings for item 19 were used. Means and standard deviations for each of these scales and subscales are provided in Table 1.

\section{Results}

Direct versus withdrawn

Stable self-directed versus stable averted (Table 2) Significantly more IA dwell time occurred in the stable self-directed condition than in the stable averted condition $(\mathrm{F}(1,43)=30.60$, $p<.001$, ES $=.42$ ). Additionally, the IA fixation count was significantly higher in the stable self-directed condition than in the stable averted condition $(\mathrm{F}(1,43)=22.23$, $p<.001$, ES = .34). These results indicate that selfdirected gaze attracted the eye more than averted gaze, as predicted. Second fixation duration was significantly longer in the stable self-directed condition than in the stable averted condition $(\mathrm{F}(1,27)=4.89, p<.05$, ES $=$ $.15)$. This difference indicates that once self-directed gaze was detected, participants returned to that interest area for continued looking time. 
Table 2 Comparison between stable averted and stable self-directed conditions. These conditions were compared to explore withdrawn and direct gaze

\begin{tabular}{|c|c|c|c|c|c|c|c|}
\hline & \multicolumn{2}{|c|}{ Stable averted } & \multicolumn{2}{|c|}{ Stable self-directed } & \multirow[b]{2}{*}{ F-value } & \multirow[b]{2}{*}{$y$} & \multirow[b]{2}{*}{ p-value } \\
\hline & M & $\mathrm{SD}$ & M & SD & & & \\
\hline IA dwell time (msec) & 236.86 & 305.72 & 994.71 & 610.70 & $(1,43)=30.6$ & .42 & $<.001$ \\
\hline IA fixation count & 1.04 & 1.21 & 3.00 & 1.58 & $(1,43)=22.23$ & .34 & $<.001$ \\
\hline IA first fixation duration (msec) & 260.85 & 190.69 & 314.24 & 95.22 & $(1,28)=1.01$ & - & 0.32 \\
\hline IA first fixation time & 1757.38 & 937.61 & 2140.94 & 1578.91 & $(1,28)=0.60$ & - & 0.44 \\
\hline IA second fixation duration (msec) & 222.69 & 60.17 & 356.63 & 211.14 & $(1,27)=4.88$ & .15 & 0.04 \\
\hline IA average fixation pupil size & 1270.77 & 431.25 & 1812.00 & 421.55 & $(1,28)=11.91$ & .30 & $<.005$ \\
\hline
\end{tabular}

Catching someone else staring

Stable averted versus delayed averted (Table 3) A similar pattern of results emerged as did between the stable averted and stable self-directed conditions. Between the stable averted condition and the delayed averted condition, we accurately predicted significantly longer IA dwell time in the delayed averted condition when compared to the stable averted condition $(\mathrm{F}(1,77)=22.89, \mathrm{p}<.001, \mathrm{ES}=.23)$ as well as more IA fixations $(\mathrm{F}(1,77)=36.42, p<.001, \mathrm{ES}=.32)$. The IA second fixation duration was significantly longer in the delayed averted condition as well $(\mathrm{F}(1,62)=5.62, p<.05, \mathrm{ES}=.08)$, which may suggest that, after initially detecting self-directed gaze (which was shifted away because that first fixation would trigger the next image), they returned to look at the IA to explore the change.

Stable self-directed versus delayed averted (Table 4) IA second fixation duration was the only significant difference found between these two conditions, with the stable self-directed condition resulting in longer looking times $(\mathrm{F}(1,65)=5.07, p<.05, \mathrm{ES}=.07)$. In the delayed averted condition, the second fixation would be on a face with averted gaze. Thus, these results suggest that self-directed gaze is more visually interesting than a shift from self-directed to averted gaze.
Being caught staring

Stable averted versus delayed self-directed (Table 5) IA dwell time and IA fixation count were, as predicted, significantly longer $(\mathrm{F}(1,74)=26.32, p<.001, \mathrm{ES}=.26)$ and higher $(\mathrm{F}(1,74)=24.15, p<.001, \mathrm{ES}=.25)$, respectively, in the delayed self-directed condition than in the stable averted condition. In the delayed self-directed condition, the second fixation would be the first fixation during which there was self-directed gaze. The IA second fixation duration was significantly longer in the delayed self-directed condition $(\mathrm{F}(1,55)=10.41, p<.005, \mathrm{ES}=.16)$, suggesting that detection of self-directed gaze resulted in longer looking times.

Delayed self-directed versus stable self-directed (Table 6) There were no significant findings in the comparison of these two conditions.

Pupil results In the conditions during which a stable condition was compared with a delayed condition, there were significant differences when the second condition ended in the opposite state (i.e., averted vs. self-directed) than the stable condition. Between the stable averted and delayed self-directed conditions, average pupil size was significantly larger in the delayed self-directed condition $(\mathrm{F}(1,55)=4.35, p<.05, \mathrm{ES}=.07)$. Pupil size was significantly larger in the stable self-directed

Table 3 Comparison between stable averted and delayed averted conditions. These conditions were compared to simulate catching someone else staring

\begin{tabular}{|c|c|c|c|c|c|c|c|}
\hline & \multicolumn{2}{|c|}{ Stable averted } & \multicolumn{2}{|c|}{ Delayed averted } & \multirow[b]{2}{*}{ F-value } & \multirow[b]{2}{*}{$y$} & \multirow[b]{2}{*}{ p-value } \\
\hline & M & $\mathrm{SD}$ & M & SD & & & \\
\hline IA dwell time (msec) & 238.86 & 305.72 & 1255.39 & 1098.37 & $(1,77)=22.89$ & .23 & $<.001$ \\
\hline IA fixation count & 1.04 & 1.2 & 3.65 & 2.11 & $(1,77)=36.42$ & .32 & $<.001$ \\
\hline IA first fixation duration (msec) & 260.85 & 190.69 & 294.22 & 88.36 & $(1,62)=.87$ & - & 0.36 \\
\hline IA first fixation time & 1757.38 & 937.61 & 2164.22 & 808.94 & $(1,62)=2.46$ & - & 0.12 \\
\hline IA second fixation duration (msec) & 222.69 & 60.17 & 277.75 & 77.85 & $(1,62)=5.62$ & .08 & 0.02 \\
\hline IA average fixation pupil size & 1270.77 & 431.25 & 1533.75 & 502.12 & $(1,62)=2.99$ & - & 0.09 \\
\hline
\end{tabular}


Table 4 Comparison between stable self-directed and delayed averted conditions. These conditions were compared to simulate catching someone else staring

\begin{tabular}{|c|c|c|c|c|c|c|c|}
\hline & \multicolumn{2}{|c|}{ Stable self-directed } & \multicolumn{2}{|c|}{ Delayed averted } & \multirow[b]{2}{*}{ F-value } & \multirow[b]{2}{*}{$y$} & \multirow[b]{2}{*}{ p-value } \\
\hline & M & $\mathrm{SD}$ & M & $\mathrm{SD}$ & & & \\
\hline IA dwell time (msec) & 994.71 & 610.70 & 1255.39 & 1098.37 & $(1,66)=.86$ & - & 0.36 \\
\hline IA fixation count & 3.00 & 1.58 & 6.65 & 2.11 & $(1,66)=1.35$ & - & 0.25 \\
\hline IA first fixation duration (msec) & 314.24 & 95.22 & 294.22 & 88.36 & $(1,66)=.63$ & - & 0.43 \\
\hline IA first fixation time & 2140.94 & 1578.91 & 2164.22 & 808.94 & $(1,66)=.01$ & - & 0.94 \\
\hline IA second fixation duration (msec) & 356.63 & 211.14 & 277.75 & 77.85 & $(1,65)=5.07$ & .07 & 0.03 \\
\hline IA average fixation pupil size & 1812.00 & 421.55 & 1533.75 & 502.11 & $(1,66)=4.22$ & .06 & 0.04 \\
\hline
\end{tabular}

condition than in the delayed averted condition as well $(\mathrm{F}(1,66)=4.22, p<.05, \mathrm{ES}=.06)$. There were no significant differences in either of the "caught someone staring" comparisons. Pupil size was significantly larger in the direct gaze condition when compared to the withdrawn condition $(\mathrm{F}(1,28)=11.91, p<.005, \mathrm{ES}=.30)$. These results suggest that self-directed gaze results in significantly larger pupil size.

Comparisons of $\mathrm{z}$-scores between the four conditions revealed a significant effect of condition on $\mathrm{z}$-score $(\mathrm{F}(3,280)=$ $5.64, p<.005, \mathrm{ES}=.06)$. Planned contrasts showed that participants experienced a significant positive increase in pupil size when images shifted from averted to direct gaze, in comparison to stable averted gaze $(t(280)=3.80, p<.005)$ and when images shifted from averted to direct gaze, versus stable direct gaze $(t(280)=2.06, p<.05)$. Condition was also found to significantly impact whether or not a significant change in pupil diameter would occur $(\mathrm{F}(3,280)=4.93$, $p<.005, \mathrm{ES}=.05)$. Planned contrasts showed that the delayed self-directed condition resulted in a significant change in pupil diameter significantly more than the delayed averted condition $(t(280)=2.16, p<.05)$, indicating that a change to self-directed gaze impacted pupil size significantly more than a change from self-directed gaze to averted. Additionally, the delayed self-directed condition resulted in a change in pupil size significantly more than the stable averted condition, indicating that pupil size changed in response to self-directed gaze $(t(280)=3.30, p<.05)$.

Behavioral measures Due to the skew of these behavioral data, natural $\log$ transformations were applied to the behavioral measures. A lack of certain types of psychopathology were a criterion for this study and this resulted in limited variability in the behavioral measures. This limited variability limits the power to identify significant relations between the behavioral measures and eye-tracking outcomes. Different variables were created for each of the variables between the conditions. For instance, the difference in fixation count between the stable averted condition and the delayed selfdirected condition was calculated for each participant. There were no significant behavioral predictors of eye-tracking outcomes.

Significant correlations are listed in Table 7. The question specifically pertaining to eye contact on the LSAS was significantly correlated with IA fixation count, indicating that more reported fear and/or avoidance was indeed associated with less direct looking at faces even in a simulated setting, such as the presentation of still images used here.

Pupil size was significantly correlated to the expressive language subscale of the SRS-ASR. Perhaps this reflects one's ability to respond with appropriate facial expressions (i.e.,

Table 5 Comparison between stable averted and delayed self-directed conditions. These conditions were compared to simulate getting caught staring

\begin{tabular}{|c|c|c|c|c|c|c|c|}
\hline & \multicolumn{2}{|c|}{ Stable averted } & \multicolumn{2}{|c|}{ Delayed self-directed } & \multirow[b]{2}{*}{ F-value } & \multirow[b]{2}{*}{$\eta$} & \multirow[b]{2}{*}{ p-value } \\
\hline & M & SD & M & SD & & & \\
\hline IA dwell time & 236.86 & 305.72 & 1429.9 & 1203.03 & $(1,74)=26.32$ & .26 & $<.001$ \\
\hline IA fixation count (msec) & 1.04 & 1.2 & 4.25 & 3.33 & $(1,74)=24.15$ & .25 & $<.001$ \\
\hline IA first fixation duration (msec) & 260.85 & 190.69 & 287.08 & 101.11 & $(1,55)=.43$ & - & 0.51 \\
\hline IA first fixation time & 1757.38 & 937.61 & 2339.95 & 1184.08 & $(1,55)=2.64$ & - & 0.11 \\
\hline IA second fixation duration (msec) & 222.69 & 60.17 & 378.27 & 169.85 & $(1,55)=10.41$ & .16 & $<.005$ \\
\hline IA average fixation pupil size & 1270.77 & 431.25 & 1582.39 & 484.54 & $(1,55)=4.35$ & .07 & 0.04 \\
\hline
\end{tabular}


Table 6 Comparison between stable self-directed and delayed self-directed conditions. These conditions were compared to simulate getting caught staring

\begin{tabular}{|c|c|c|c|c|c|c|c|}
\hline & \multicolumn{2}{|c|}{ Stable self-directed } & \multicolumn{2}{|c|}{ Delayed self-directed } & \multirow[b]{2}{*}{ F-value } & \multirow[b]{2}{*}{$\eta$} & \multirow[b]{2}{*}{ p-value } \\
\hline & M & SD & M & SD & & & \\
\hline IA dwell time (msec) & 994.71 & 610.70 & 1429.90 & 1203.03 & $(1,63)=2.02$ & - & 0.16 \\
\hline IA fixation count & 3.00 & 1.58 & 4.25 & 3.33 & $(1,63)=2.20$ & - & 0.14 \\
\hline IA first fixation duration (msec) & 314.24 & 95.22 & 287.05 & 101.11 & $(1,59)=.92$ & - & 0.34 \\
\hline IA first fixation time & 2140.94 & 1578.91 & 2339.95 & 1184.08 & $(1,59)=.29$ & - & 0.60 \\
\hline IA second fixation duration (msec) & 356.63 & 211.14 & 378.27 & 169.85 & $(1,58)=.17$ & - & 0.68 \\
\hline IA average fixation pupil size & 1812.00 & 421.55 & 1582.39 & 484.54 & $(1,59)=2.95$ & - & 0.09 \\
\hline
\end{tabular}

with interest when self-directed gaze is detected) is related to one's ability to respond appropriately verbally (i.e., via expressive language).

\section{Discussion}

The purpose of this study was to develop a paradigm to study the stare-in-the-crowd effect in stable and dynamic settings. To achieve this goal, a new paradigm using images with a crowd of people was presented in an effort to more closely approximate actual social setting. The experiences of direct gaze, withdrawn gaze, being caught staring and catching someone else staring were examined by examining gaze behavior. In an effort to explore the relationship between social perception and behavioral phenotypes, behavioral measures were also administered.

This paradigm did, in fact, capture this stare-in-the-crowd effect. In comparing the IA dwell time, fixation count, fixation durations, and fixation times, we quantitatively showed that self-directed gaze attracts the eye of viewers even when surrounded by a number of other faces. Self-directed gaze resulted in significantly more looking time. This result, in and of itself, is not surprising because the detection of selfdirected gaze is known to be a strength of human perception. In fact, detection of self-directed gaze develops within days of birth (Farroni et al., 2002). In line with these findings, eventrelated potential (ERP) and functional magnetic resonance

Table 7 Significant correlations between behavioral measures and eyetracking outcomes

\begin{tabular}{cllll}
\hline & & $\begin{array}{l}\text { Expressive } \\
\text { subscale of } \\
\text { SRS }\end{array}$ & $\begin{array}{l}\text { LSAS19 } \\
\text { Fear }\end{array}$ & $\begin{array}{l}\text { LSAS19 } \\
\text { Avoid }\end{array}$ \\
\hline $\begin{array}{c}\text { IA average } \\
\text { pupil size }\end{array}$ & $\begin{array}{l}\text { Pearson's Correlation } \\
\text { Sig (2-tailed) }\end{array}$ & 0.53 & $n s$ & $n s$ \\
$\begin{array}{c}\text { IA fixation } \\
\text { count }\end{array}$ & Pearson's Correlation & $n s$ & -0.45 & -0.49 \\
\hline
\end{tabular}

imaging (fMRI) studies have demonstrated that more attention is allocated to self-directed gaze because more resources are necessary for direct gaze processing (Conty, N'Diaye, Tijus, \& George, 2007; Dalton et al., 2005). Instead of using one set of eyes in the visual stimuli as is common in the literature, including an image of a group of people allowed us to quantify how changes in gaze detection affected gaze behavior when there were multiple possible gaze targets. Even with other people to look at, it was still self-directed gaze that attracted the most visual attention.

The significant findings in gaze behavior showed that shifts in self-directed gaze are registered, as evidenced by increased looking, in response to an individual's own gaze. Given the many social rules about looking (e.g., "Don't stare!" and "Look at me when I am speaking to you"), there are numerous presentations of different gaze scenarios that have yet to be explored in relation to different types of psychopathology. Furthermore, given the early development of gaze behaviors, more nuanced visual paradigms might have utility in early screening for disorders before they manifest behaviorally.

The significant differences on the second fixation outcomes and not the first are of interest. Across comparisons in the results above (except when the final image was selfdirected in both conditions being compared), these significant findings indicate that the second fixation reflects visual interest in self-directed gaze. There is evidence that initial scanning of visual stimuli is simply sampling from the whole; saccades (series of short fixations) are the human tool to perceive a large amount of information and to process only the most salient aspects. Thus, an initial fixation may be occurring in our initial scan and then an inner mechanism is activated by the detection of self-directed gaze. This would then draw our eye back to this area, resulting in shifts in gaze direction being captured by significant differences on the second fixation outcomes. Thus, a lack of differences between the conditions for the first fixation outcomes follows logically. Moving forward, time between the first and second fixation could help further explore characteristics of the change from automatic to controlled perception of gaze shifts. 
Overall, the presence of direct gaze resulted in a significantly greater pupil size increase than averted gaze and changes in images from averted gaze to direct gaze resulted in the most significant increases, even above changes from direct gaze to averted gaze. Thus, while a change of gaze resulted in increase pupil size, a change in the direction of self-directed gaze resulted in the largest change. Characterizing the changes in pupil diameter relative to dynamic visual stimuli will be useful in understanding what social changes elicit parasympathetic and sympathetic activity. These reactions could provide clues in future studies from nonverbal populations, or populations with low insight into their own reactions.

In this study, the behavioral data did not correlate as strongly with gaze behavior as initially anticipated. There are two possible reasons for this. The first is that this reflects the lack of relationship between behavioral phenotypes and gaze behavior in the stare-in-the-crowd effect. Other perceptual behaviors, such as the attributional bias and irregular facial perception patterns, have been found to be significantly correlated with externalizing and internalizing symptoms previously (Horley, Williams, Gonsalvez, \& Gordon, 2004; Ross, Harris, Olincy, \& Radant, 2000; Steinberg \& Dodge, 1983).Thus, it seems unlikely that this relationship between behavioral phenotypes and gaze behavior would be completely nonexistent. The second possibility is that the variability in this sample, by design, was limited across the behavioral measures. Thus, given the low rate of psychopathology, it is possible the limited variability on the measures as well as the relative homogeneity of the gaze behaviors masked possible relationships between the two. This is evident in the fact that there were correlations with key behavioral variables, but not so much so that, at their extremes, they were associated with the gaze measures. In future studies, we hope to further explore these relations as we study clinical populations.

The novelty of these stimuli may be a limitation of this study. However, given the significant results in the gaze behaviors, it seems that these images successfully captured the effects we were attempting to recreate. Furthermore, the significant results all had medium to large effect sizes (Cohen, 1988), indicating robust effects of condition, even with a sample of only 35 .

Looking ahead, the goal after establishing the utility of these images to capture the stare-in-the-crowd effect is twofold; first, to use this paradigm to explore social abilities across dimensions of psychopathology and developmental stages, and second, to combine with other psychophysiological monitoring systems to develop a more comprehension model of these phenomena. These models are vital to informing intervention design and timing of implementation. Gaze detection is a piece of the larger social interaction domain and success in this area is critical for adaptive functioning. Success in social interactions more broadly has implications for psychopathology, quality of friendships, and work success later in life (Baron \& Markman, 2000). Peer rejection predicts both aggressive and non-aggressive behaviors throughout childhood (Deater-Deckard, 2001), while the presence of even a single best friend serves a protective factor against these behaviors, even in the face of harsh parenting practices at home (Schwartz, Dodge, Pettit, \& Bates, 2000). A lack of peer support is associated with loneliness (Boivin, Hymel, \& Bukowski, 1995) and the risk of substance abuse is lower in childhood and adolescence when the child has a friend (Newcomb \& Bentler, 1988). Thus, from the knowledge gained in future studies, we may be able to best assist those struggling with social perceptual deficits.

In sum, the goal of developing a set of visual stimuli and a paradigm to capture the many facets of the SITC effect was achieved. Although no significant behavioral correlates were detected, the gaze behavior and pupil dilation that was captured during the course of this study provide baseline profiles for typical responses to direct and withdrawn gaze, being caught staring, and catching others staring. With continued applications of this paradigm and technological advances of eye-tracking technology, future results will allow for development of even more sophisticated methods of building on the study of specific components of the SITC effect.

Acknowledgments Ms. Crehan has no biomedical financial interests or potential conflicts of interest to report. Dr. Robert R. Althoff receives grant or research support from the National Institute of Mental Health (NIMH), the National Institute of General Medical Sciences (NIGMS), and the Klingenstein Third Generation Foundation. This research was supported in part by Center of Biomedical Research Excellence (COBRE) Center for Neuroscience Excellence Award P30GM103498/ P30RR032135 and COBRE award P20GM103644 from NIGMS along with K08MH082116 from NIMH. The content is solely the responsibility of the authors and does not necessarily represent the official views of NIGMS, NIMH, or the National Institutes of Health (NIH). The authors wish to thank their research team, especially Samantha Holland, Sara Mahon, Joe Nowlan, and Asher Wiley for their efforts on this project.

\section{References}

Achenbach, T. M. (2009). The achenbach system of empirically based assessemnt (aseba): Development, findings, theory, and applications. Burlington: University of Vermont Research Center for Children, Youth and Families.

Baron, R.A., \& Markman, G.D. (2000). Beyond social capital: How social skills can enhance entrepreneurs' success. The Academy of Management Executive, 14(1).

Beatty, J. (1982). Task-evoked pupillary responses, processing load, and the structure of processing resources. Psychological Bulletin, 91(2), 276-292.

Boivin, M., Hymel, S., \& Bukowski, W. M. (1995). The roles of social withdrawal, peer rejection, and victimization by peers in predicting loneliness and depressed mood in childhood. Development and Psychopathology, 7(4), 765-785.

Bolte, S. (2012). Brief report: The social responsiveness scale for adults (srs-a): Initial results in a german cohort. Journal of Autism and 
Developmental Disorders, 42(9), 1998-1999. doi:10.1007/s10803011-1424-5

Breed, G. (1972). The effect of intimacy: Reciprocity or retreat? British Journal of Social and Clinical Psychology, 11(2), 135-142.

Cohen, J. (1988). Statistical power analysis for the behavioral sciencies (2nd ed.). Hillside, NJ: Lawrence Erlbaum Associates, Publishers.

Constantino, J. N., \& Gruber, C. P. (2005). The Social Responsiveness Scale (SRS) manual. Los Angeles: Western Psychological Services.

Conty, L., N'Diaye, K., Tijus, C., \& George, N. (2007). When eye creates the contact! ERP evidence for early dissociation between direct and averted gaze motion processing. Neuropsychologia, 45(13), 3024 3037.

Cook, M., \& Smith, J. M. (1975). The role of gaze in impression formation. British Journal of Social and Clinical Psychology, 14(1), 19-25.

Dalton, K. M., Nacewicz, B. M., Johnstone, T., Schaefer, H. S., Gernsbacher, M. A., Goldsmith, H. H., \& Davidson, R. J. (2005). Gaze fixation and the neural circuitry of face processing in autism. Nature Neuroscience, 8(4), 519-526.

Deater-Deckard, K. (2001). Annotation: Recent research examining the role of peer relationships in the development of psychopathology. Journal of Child Psychology and Psychiatry, 42(5), 565-579.

Farroni, T., Csibra, G., Simion, F., \& Johnson, M. H. (2002). Eye contact detection in humans from birth. Proceedings of the National Academy of Science U S A, 99(14), 9602-9605.

Fletcher-Watson, S., Leekam, S. R., Benson, V., Frank, M. C., \& Findlay, J. M. (2009). Eye-movements reveal attention to social information in autism spectrum disorder. Neuropsychologia, 47(1), 248-257. doi:10.1016/j.neuropsychologia.2008.07.016

Heimberg, R. G., Horner, K. J., Juster, H. R., Safren, S. A., Brown, E. J., Schneier, F. R., \& Liebowitz, M. R. (1999). Psychometric properties of the liebowitz social anxiety scale. Psychological Medicine, 29(1), 199-212.

Horley, K., Williams, L. M., Gonsalvez, C., \& Gordon, E. (2004). Face to face: Visual scanpath evidence for abnormal processing of facial expressions in social phobia. Psychiatry Research, 127(1-2), 43-53. doi:10.1016/j.psychres.2004.02.016

Jellison, J. M., \& Ickes, W. J. (1974). The power of the glance: Desire to see and be seen in cooperative and competitive situations. Journal of Experimental Social Psychology, 10, 444-450.

Kelly, E. W. (1978). Effects of counselor's eye contact on student-clients' perceptions. Perceptual and Motor Skills, 46, 627-632.

Kendon, A. (1967). Some functions of gaze-direction in social interaction. Acta Psychologica, 26(1), 22-63.

Kleinke, C. L. (1986). Gaze and eye contact: A research review. Psychological Bulletin, 100(1), 78-100.

Klin, A., Jones, W., Schultz, R., Volkmar, F., \& Cohen, D. (2002). Visual fixation patterns during viewing of naturalistic social situations as predictors of social competence in individuals with autism. Archives of General Psychiatry, 59(9), 809-816.

Knapp, M. L., Hart, R. P., Friedrich, G. W., \& Shulman, G. M. (1973). The rhetoric of goodbye: Verbal and nonverbal correlates of human leave-taking. Speech Monographs, 40(3).

Levine, M. H., \& Sutton-Smith, B. (1973). Effects of age, sex and task on visual behaviora during dyadic interactions. Developmental Psychology, 9, 400-405.

Macrae, C. N., \& Bodenhausen, G. V. (2000). Social cognition: Thinking categorically about others. Annual Review of Psychology, 51, 93 120. doi:10.1146/annurev.psych.51.1.93

Macrae, C. N., Hood, B. M., Milne, A. B., Rowe, A. C., \& Mason, M. F. (2002). Are you looking at me? Eye gaze and person perception. Psychological Science, 13(5), 460-464.

McKelvie, S. J. (1976). The role of eyes and mouth in the memory of a face. American Journal of Psychology, 89, 311-323.

Newcomb, M. D., \& Bentler, P. M. (1988). Impact of adolescent drug use and social support on problems of young adults: A longitudinal study. Journal of Abnormal Psychology, 97(1), 64-75.

Otteson, J. P., \& Otteson, C. R. (1980). Effects of teacher's gaze on children's story recall. Perceptual and Motor Skills, 50, 35-42.

Preuschoff, K., Hart, B. M., \& Einhauser, W. (2011). Pupil dilation signals surprise: Evidence for noradrenaline's role in decision making. Journal of Frontal Neuroscience, 5, 115.

Ross, R. G., Harris, J. G., Olincy, A., \& Radant, A. (2000). Eye movement task measures inhibition and spatial working memory in adults with schizophrenia, adhd, and a normal comparison group. Psychiatry Research, 95(1), 35-42.

Schwartz, D., Dodge, K. A., Pettit, G. S., \& Bates, J. E. (2000). Friendship as a moderating factor in the pathway between early harsh home environment and later victimization in the peer group. The conduct problems prevention research group. Developmental Psychology, 36(5), 646-662.

Steinberg, M. S., \& Dodge, K. A. (1983). Attributional bias in aggressive adolescent boys and girls. Journal of Social and Clinical Psychology, 1(4), 312-321.

Steinhauer, S. R., Siegle, G. J., Condray, R., \& Pless, M. (2004). Sympathetic and parasympathetic innervation of pupillary dilation during sustained processing. International Journal of Psychophysiology, 52(1), 77-86. doi:10.1016/j.ijpsycho.2003.12.005

von Grunau, M., \& Anston, C. (1995). The detection of gaze direction: A stare-in-the-crowd effect. Perception, 24(11), 1297-1313.

Walker-Smith, G. J., Gale, A. G., \& Findlay, J. M. (1977). Eye movement strategies involved in face perception. Perception, 6(3), 313-326.

Wheeler, R. W., Baron, J. C., Michell, S., \& Ginsburg, H. J. (1979). Eye contact and the perception of intelligence. Bulletin of the Psychonomic Society, 13, 101-102.

Yarbus, A.L. (1967). Eye movements during perception of complex objects. Eye movements and vision (pp. 171-211): Springer. 